\title{
Naphthalimide Analogue UNBS5162
}

National Cancer Institute

\section{Source}

National Cancer Institute. Naphthalimide Analogue UNBS5162. NCI Thesaurus. Code C102850.

An amonafide (naphthalimide) derivative and pan-antagonist of chemokine lig and (CXCL) expression, with potential anti-ang iogenic activity. Although UNBS5162 is a derivative of amonafide, this agent appears to have a different profile to that of amonafide and its exact mechanism of action remains to be fully elucidated. This agent seems to decrease the expression of various proang iogenic CXCL chemokines in vitro and may have synergistic effects with radiotherapy or chemotherapy. CXCLs are small cytokines in the CXC chemokine family that are overexpressed in certain cancers; CXCL-mediated signaling plays a key role in angiogenesis and tumor progression. 